\title{
Relationship between markers of endothelial dysfunction and inflammation and subclinical atherosclerosis in HIV-infected male patients below 55 years of age
}

\author{
Mateja Pirs ${ }^{1}$, Borut Jug ${ }^{2}$, Barbara Eržen², Mišo Šabović ${ }^{2}$, Primož Karner³, Mario Poljak ${ }^{1}$, Janez Tomažič3
}

\begin{abstract}
Introduction: Antiretroviral therapy in HIV-infected patients appears to be associated with increased incidence of cardiovascular disease (CVD). The aim of our study was to investigate the differences in markers of inflammation, endothelial dysfunction and prothrombotic state between treated and untreated HIV-infected patients with or without subclinical atherosclerosis.

Methods: Eighty-six Slovenian HIV-infected male patients below the age of 55 participated in our study. Levels of high-sensitivity Creactive protein (hsCRP), vascular cell adhesion molecule 1 (VCAM-1) and plasminogen activator inhibitor 1 (PAl-1) were measured. The presence of subclinical atherosclerosis was determined by measuring carotid intima-media thickness.

Results: The level of hsCRP was significantly increased in HIV-infected patients; it was higher in treated than untreated patients. VCAM-1 was significantly increased; it was higher in untreated than treated patients. PAI-1 was significantly increased; there were no differences between untreated and treated patients. Patients with subclinical atherosclerosis had elevated hsCRP; levels of VCAM-1 and PAI-1 were not significantly different.

Conclusion: Signs of systemic and vascular inflammation persist in both untreated and treated HIV infected patients. None of the studied markers contributed to improved assessment of subclinical atherosclerosis. The usefulness of such markers in routine clinical evaluation of CVD risk in HIV infected patients remains unclear.
\end{abstract}

Keywords: HIV infection, endothelial dysfunction, subclinical atherosclerosis, hsCRP, VCAM-1, PAI-1

Received: 12 July 2014 | Returned for modification: 28 July 2014 | Accepted: 11 August 2014

\section{Introduction}

Combination antiretroviral treatment (cART) has led to markedly increased life expectancy in HIV infected individuals. For patients who have access to cART and are motivated to adhere to the treatment regime HIV has become a chronic disease (1). Increased life expectancy is coupled with increased burden of various chronic conditions, among them cardiovascular disease (CVD). The reasons for increased CVD risk among this patient population and the extent to which different factors such as HIV-related differences in traditional risk factors, HIV infection itself, or the use of cART contribute to this risk remain controversial $(2,3)$. Pathogenesis of atherosclerosis in HIV infection is complex as HIV infection induces chronic inflammation which persists even after the initiation of cART, and the use of cART itself leads to metabolic complications. In treatment naïve patients inflammatory factors and HIV infection probably play a more significant role, while in patients treated with cART the effect of metabolic factors may become more pronounced (4). HIV infection itself induces chronic systemic inflammation and immune activation, resulting in elevations of various biologic markers associated with CVD risk in HIV negative populations $(5,6)$. Inflammatory response to HIV infection leads to endothelial activation and release of vascular adhesion molecules as well as an increase in serum levels of different inflammatory markers. Endothelial dysfunction represents early qualitative changes in atherogenesis (7). Chronic systemic inflammation may contribute to increased incidence of CVD as some inflammatory markers (e.g. CRP, IL-6, ICAM-1, etc.) have been shown to correlate with and may even predict CVD in HIV infected and uninfected individuals $(5,6,8,9)$. Impaired fibrinol- ysis and prothrombotic state are independent risk factors for CVD. Elevated levels of PAI-1 are associated with insulin resistance in general as well as in HIV infected population (10).

A national study of the prevalence of and risk factors for CVD in Slovenian HIV infected patients has shown increased prevalence of subclinical atherosclerosis in HIV infected patients below the age of 55. The aim of this part of the study was to evaluate markers of inflammation, endothelial dysfunction and prothrombotic state and investigate their correlation with the presence of subclinical atherosclerosis in Slovenian HIV infected patients below the age of 55 .

\section{Patients and Methods}

A prospective cross-sectional national study was performed at the Department of Infectious Diseases, University Medical Centre Ljubljana (UMCL), Slovenia. The investigation was approved by the institutional Ethics Committee for clinical research.

\section{Patients}

86 consecutive HIV infected male patients were included in the study; patients lacking all measurements of markers of inflammation, endothelial dysfunction and prothrombotic state were excluded from this analysis; in total $82 \mathrm{HIV}$ infected male patients were included. Inclusion criteria were HIV infection for more than one year, male gender and age below 55 years; the study population was described in detail elsewhere (11). Patients' history was recorded; physical examination and routine laboratory tests were performed on all subjects. Use of cART, duration of treatment and

${ }^{1}$ Institute of Microbiology and Immunology, Faculty of Medicine, University of Ljubljana, Slovenia. ${ }^{2}$ Department of Vascular Diseases, University Medical Centre Ljubljana, Ljubljana, Slovenia. ${ }^{3}$ Clinic for Infectious Diseases and Febrile Illnesses, University Medical Centre Ljubljana, Ljubljana, Slovenia.

$\bowtie$ Corresponding author: mateja.pirs@mf.uni-lj.si 
type of cART was noted (protease inhibitor (PI) based or non-nucleoside reverse transcriptase inhibitor (NNRTI) based treatment regime). We have compared our results with the results from historical HIV negative control group of 25 healthy men below the age of 55 (12).

\section{Markers of inflammation and vascular activationt}

Markers of inflammation and vascular activation were measured using commercially available kits according to the manufacturer's instructions. High sensitivity $\mathrm{C}$ reactive protein (hsCRP) was measured using CardioPhase hsCRP test (Siemens, Germany). Vascular cell adhesion molecule 1 (VCAM-1) was measured using Quantikine ELISA Human sVCAM-1/CD106 Immunoessay (R\&D Systems, Inc., United Kingdom). Plasminogen activator inhibitor 1 (PAI-1) was measured using TriniLIZE PAI-1 Activity (Trinity Biotech, Ireland).

\section{Carotid intima media thickness measurement}

Carotid intima media thickness (CIMT) was assessed by the B mode high resolution ultrasound technique (Diasonics VST ultrasound system) as described previously $(13,14)$. Mean CIMT was calculated as the average of three measurements of the left and right carotid artery. Increased CIMT was defined as more than 0.8 $\mathrm{mm}$ thick carotid intima media $\left(\mathrm{CIMT}_{>0.8 \mathrm{~mm}}\right)(15,16)$. Plaque was defined as described previously (17).

\section{CVD risk assessment}

Systematic Coronary Risk Evaluation (SCORE) is based on gender, age, systolic blood pressure, smoking status and total cholesterol/ HDL cholesterol ratio. As Slovenia is among low risk regions of Europe, the risk chart for low-risk countries was used. The patients were classified as having low $(<1 \%)$, medium ( $\geq 1$-to $<5 \%)$, high ( $\geq 5$-to $<10 \%$ ) or very high $(\geq 10 \%)$ cardiovascular risk (18).

\section{Statistical analysis}

Statistical analysis was performed using statistical software IBM SPSS Statistics version 20.0 (IBM corp., NY, USA). We used Mann Whitney U-test or Kruskall-Wallis test to compare continuous var- iables and Fisher's exact test or chi-squared test for categorical variables. Logistic regression was used to determine the influence of markers of inflammation, endothelial dysfunction and prothrombotic state, SCORE (combined influence of classical cardiovascular risk factors), use of PI or NRTI, CD4 cell count and viral load on $\mathrm{CIMT}_{>0.8 \mathrm{~mm}}$ and presence of carotid plaques.

\section{Results}

The main baseline characteristics of the study population were described in detail elsewhere (11). The mean age of HIV infected study population was 39 years, $70 \%$ were on cART with mean exposure of 4.8 years.

Markers of inflammation, endothelial dysfunction and prothrombotic state in HIV infected patients and HIV negative patients are shown in Table 1. We have found significantly elevated level of hsCRP among HIV infected patients compared to HIV negative controls ( $\mathrm{p}$ o.015). hsCRP levels were significantly higher in treated than in treatment naïve patients ( $\mathrm{p}$ o.031). There were no statistically significant differences based on the type of cART (PI or NRTI based) ( $\mathrm{p}$ o.082). There was no correlation with CIMT $_{>0.8 \mathrm{~mm}}$ or the presence of plaque. We have noted a correlation between hsCRP level and viral load at the time of the study ( $\mathrm{p}$ 0.009). We have found significantly elevated levels of VCAM-1 among HIV infected patients compared to HIV negative controls ( $p$ o.ooo). VCAM-1 levels were significantly higher in treatment-naïve than in treated patients ( $\mathrm{p}$ o.036). There were significant differences based on the type of cART (PI or NRTI based) (568.2 vs. 425.0, $p$ o.033). There was no correlation with CIMT $_{>0.8 \mathrm{~mm}}$ or the presence of plaque. We have noted correlation between hsCRP level and the duration of HIV infection ( $p$ o.006) and CD4 cell count ( $p$ o.ooo). We have found significantly elevated level of PAI-1 among HIV infected patients compared to HIV negative controls ( $\mathrm{p}$ o.00o). There were no significant differences in PAI-1 levels between treatment naïve and treated HIV infected patients ( $\mathrm{p}$ 0.903) or type of cART (PI or NRTI based) (p o.o94).

Comparison of markers of inflammation, endothelial dysfunction and prothrombotic state and CIMT measurements is shown in Table 2. HIV infected patients with subclinical atherosclerosis (as defined by $\mathrm{CIMT}_{>0.8 \mathrm{~mm}}$ ) had statistically significantly higher levels of hsCRP ( $\mathrm{p}$ 0.04), in contrast the differences in serum levels of VCAM-1 and PAI-1 were not statistically significant. In the logistic

Table 1 | Markers of inflammation, endothelial dysfunction and prothrombotic state in HIV infected patients and HIV negative patients.

\begin{tabular}{|c|c|c|c|c|}
\hline & \multirow{2}{*}{ HIV-negative patients } & \multicolumn{3}{|c|}{ HIV infected patients } \\
\hline & & All & Treatment-naïve & Treated group \\
\hline No. of patients & 25 & 82 & 24 & 58 \\
\hline hsCRP (mg/ml) & $1.458(0.287)$ & $3.21(3.97)$ & $2.26(0.589)$ & $3.59(0.565)$ \\
\hline VCAM-1 (ng/ml) & $286.53(12.14)$ & $505.64(305.71)$ & $532.93(41.83)$ & $494.17(44.88)$ \\
\hline PAI-1 (IU/ml) & $10.26(1.46)$ & $42.25(25.76)$ & $41.77(5.84)$ & $41.63(3.22)$ \\
\hline
\end{tabular}

Values are shown as medians and standard deviation.

hsCRP - high-sensitivity C-reactive protein, VCAM-1 - vascular cell adhesion molecule 1, PAI-1 - plasminogen activator inhibitor 1 , IU - international units.

Table 2 | Markers of inflammation, endothelial dysfunction and prothrombotic state in HIV infected patients with and without subclinical atherosclerosis as defined by $\mathrm{CIMT}_{>0.8 \mathrm{~mm}}$.

\begin{tabular}{lccc}
\hline & HIV-infected patients (all) & \multicolumn{2}{c}{ Subclinical atherosclerosis } \\
\cline { 3 - 4 } & & No value & Yes \\
\hline No. of patients & 82 & 41 & 41 \\
hsCRP $(\mathrm{mg} / \mathrm{ml})$ & $3.21(3.97)$ & $3.13(0.58)$ & $3.29(0.65)$ \\
VCAM-1 $(\mathrm{ng} / \mathrm{ml})$ & $505.64(305.71)$ & $494.79(53.55)$ & $517.00(41.75)$ \\
PAl-1 (IU $/ \mathrm{ml})$ & $42.25(25.76)$ & $41.55(4.49)$ & 0.04 \\
\hline
\end{tabular}

Values are shown as medians and standard deviation.

hsCRP - high-sensitivity C-reactive protein, VCAM-1 - vascular cell adhesion molecule 1, PAI-1 - plasminogen activator inhibitor 1 , IU - international units. 

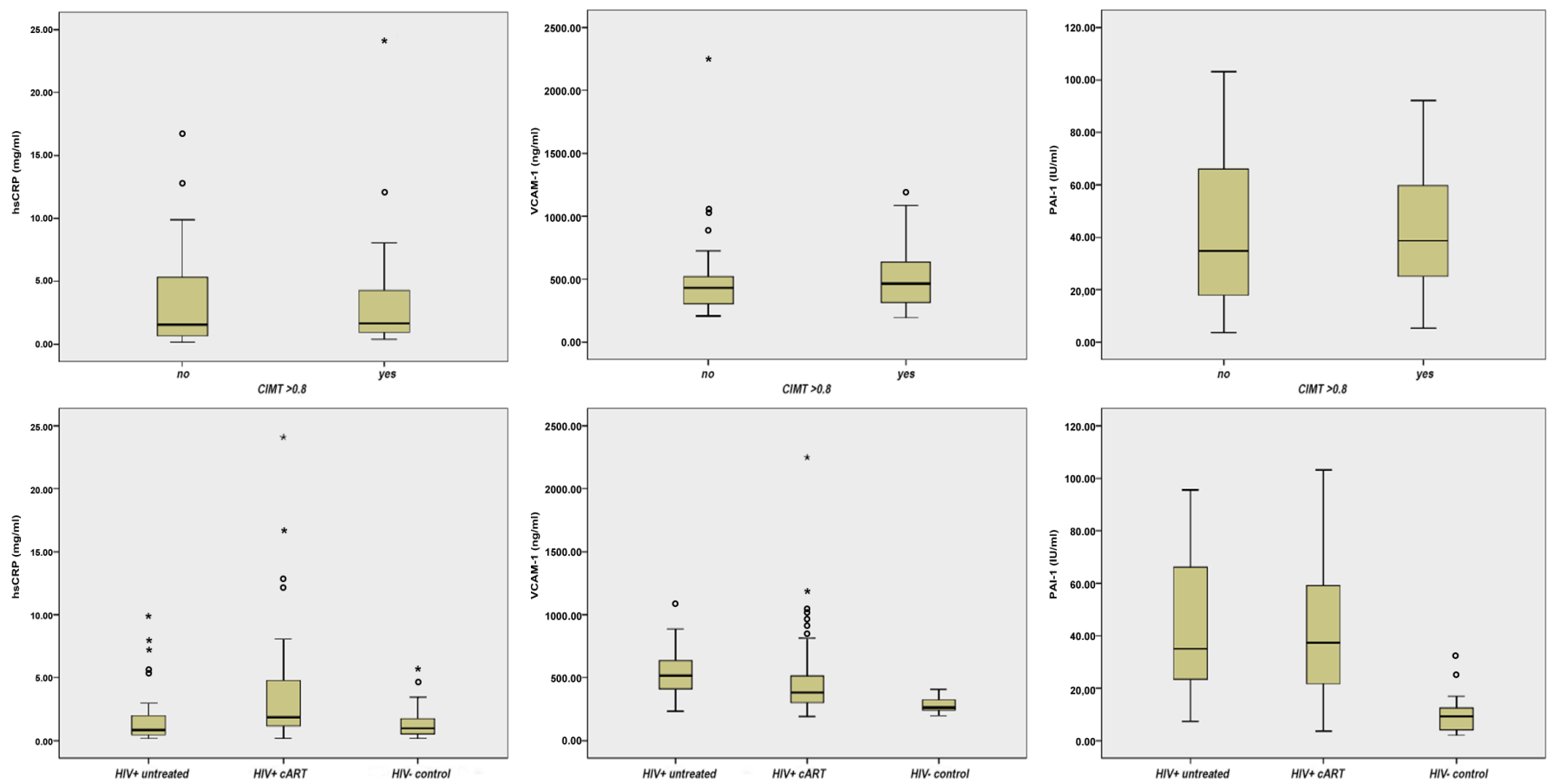

Figure 1 | Markers of inflammation endothelial dysfunction and prothrombotic state in HIV infected patients with and without subclinical atherosclerosis as defined by $\mathrm{CIMT}_{20.8 \mathrm{~mm}}$. hSCRP - high-sensitivity C-reactive protein, VCAM-1 - vascular cell adhesion molecule 1, PAI-1 - plasminogen activator inhibitor 1, IU - international units.

regression analysis where the dependent variable was CIMT $_{>0.8 \mathrm{~mm}}$ SCORE (p o.002) was the only variable that remained in the model. Similarly SCORE ( $\mathrm{p}$ o.026) was the only variable that remained in the model when dependent variable was the presence of plaque.

\section{Discussion}

HIV infection results in chronic systemic immune activation and elevated serum levels of inflammatory cytokines (19, 20). Reduction of serum levels of markers of endothelial dysfunction (e.g. ICAM-1, VCAM-1, selectin E, etc.) were noted following the introduction of cART, however serum levels remained elevated compared to HIV negative controls $(21,22)$.

We have found elevated levels of hsCRP in HIV infected patients compared to healthy controls. Similar results were found in several other studies $(23,24)$. There were however some studies that found no significant differences in levels of hsCRP between HIV infected patients and healthy controls (25). Treated patients (regardless of PI- or NNRTI-based regimes) had higher levels of hsCRP than treatment-naïve patients, similar results were reported by Lebech et al. (26). We have found no correlation between CIMT $_{>0.8 \mathrm{~mm}}$ or the presence of plaques, we did, however, find a correlation between a level of hsCRP and viral load at the time of the study. Falcao et al. have similarly noted higher incidence of subclinical atherosclerosis among HIV infected patients, but they did not find any correlation between markers of inflammation or endothelial dysfunction and subclinical atherosclerosis (27). Triant et al. have, however, noted that HIV infected patients with increased CRP had a markedly increased relative risk of acute myocardial infarction (28). Similar findings were reported in several other studies $(29,30)$.

We have found significantly higher levels of VCAM-1 in HIVinfected patients than healthy controls. Wolf et al. have also found elevated levels of VCAM-1 among treatment-naïve patients with reduction of VCAM-1 level after the introduction of CART; it, however, remained higher than in healthy controls (21). Similar results were found in some other studies (31-34). In contrast, Guz- man Fulgencio et al. did not find any statistically significant differences between HIV infected patients and healthy controls (35).

We have also found significantly higher levels of PAI-1 in HIV infected patients than in healthy controls. Similar results were reported in several other studies $(26,34)$. We have found no differences between treatment-naïve and treated HIV infected patients (regardless of the type of cART). Contrary to our findings Koppel et al. have found higher levels of PAI-1 in patients whose treatment regime included PI (36).

When levels of markers of inflammation, endothelial dysfunction and prothrombotic state were compared in HIV infected patients with and without subclinical atherosclerosis, we have found significantly higher levels of hsCRP in patients with CIMT $_{>0.8 \mathrm{~mm}}$. When we investigated the influence of different parameters on the presence of $\mathrm{CIMT}_{>0.8 \mathrm{~mm}}$ or carotid plaques using logistic regression model, only SCORE CVD risk algorithm remained in the model.

Various studies have demonstrated that HIV infection results in chronic systemic immune activation, endothelial dysfunction and prothrombotic state; and while these findings may contribute to the understanding of underlying pathogenesis, the usefulness of such markers in routine clinical evaluation of the patients and prevention of CVD is unclear. hsCRP has been the most widely evaluated marker. Even in healthy populations various confounders influence the levels of hsCRP such as classical CVD risk factors and - most importantly for HIV-infected populations - the potential presence of low grade inflammatory diseases (18). Centers for Disease Control/American Heart Association guidelines do recommend measuring hsCRP in patients with moderate risk of CVD (10\% to $20 \%$ risk of CVD over 10 years) to identify individuals who should be considered for intensified medical therapy and preventative measures (37). This approach has not been validated in HIV infected patients (30).

\section{Conclusion}

HIV infection results in chronic systemic immune activation and elevated serum levels of inflammatory cytokines. Our study has 
demonstrated significantly increased levels of hsCRP in HIV infected patients with subclinical atherosclerosis, in contrast no significant differences in levels of VCAM-1 or PAI-1 were found. None of the studied markers of inflammation, endothelial dys-

\section{References}

1. Deeks SG, Lewin SR, Havlir DV. The end of AIDS: HIV infection as a chronic disease. Lancet. 2013;382:1525-33.

2. Grunfeld C, Kotler DP, Arnett DK, Falutz JM, Haffner SM, Hruz P, et al. Contribution of metabolic and anthropometric abnormalities to cardiovascular disease risk factors. Circulation. 2008;118:e20-8.

3. Delaney JA, Scherzer R, Biggs ML, Shliplak MG, Polak JF, Currier JS, et al. Associations of antiretroviral drug use and HIV-specific risk factors with carotid intima-media thickness. AIDS. 2010;24:2201-9.

4. Piconi S, Parisotto S, Rizzardini G, Passerini S, Meraviglia P, Schiavini M, et al. Atherosclerosis is associated with multiple pathogenic mechanisms in HIV-infected antiretroviral-naive or treated individuals. AIDS. 2013;27:381-9.

5. Fichtenbaum CJ. Inflammatory markers associated with coronary heart disease in persons with HIV infection. Curr Infect Dis Rep. 2010;13:94-101.

6. Blake GJ, Ridker PM. Inflammatory bio-markers and cardiovascular risk prediction. J Intern Med. 2002;252:283-94.

7. Libby P, Ridker PM, Hansson GK. Progress and challenges in translating the biology of atherosclerosis. Nature. 2011;473:317-25.

8. Rönsholt FF, Ullum H, Katzenstein TL, Gerstoft J, Ostrowski SR. Persistent inflammation and endothelial activation in HIV-1 infected patients after 12 years of antiretroviral therapy. PLoS ONE. 2013;8:e65182.

9. López M, San Román J, Estrada V, Vispo E, Blanco F, Soriano V. Endothelial dys function in HIV infection--the role of circulating endothelial cells, microparticles, endothelial progenitor cells and macrophages. AIDS Rev. 2012;14:223-30.

10. Hadigan C, Meigs JB, Rabe J, D'Agostino RB, Wilson PW, Lipinska I, et al. Increased PAI-1 and TPA antigen levels are reduced with metformin therapy in HIV-infected patients with fat redistribution and insulin resistance. J Clin Endocr Metab. 2001;86:939-43.

11. Pirš M, Erzen B, Sabovic M, Karner P, Vidmar L, Poljak M, et al. Early atherosclerosis in HIV-infected patients below the age of 55 years: Slovenian national study. Wien Klin Wochenschr. 2014;126:263-9.

12. Erzen B, Sabovic M, Sebestjen M, Poredos P. Endothelial dysfunction, intimamedia thickness, ankle-brachial pressure index, and pulse pressure in young post-myocardial infarction patients with various expressions of classical risk factors. Heart Vessels. 2007;22:215-22.

13. Mulligan K, Grunfeld C, Tai VW, Algren H, Pang M, Chernoff DN, et al. Hyperlipidemia and insulin resistance are induced by protease inhibitors independent of changes in body composition in patients with HIV infection. J Acquir Immune Defic Syndr. 2000;23:35-43.

14. Pignoli P, Tremoli E, Poli A, Oreste P, Paoletti R. Intimal plus medial thickness of the arterial wall: a direct measurement with ultrasound imaging. Circulation. 1986;74:1399-406.

15. O'Leary DH, Polak JF, Kronmal RA, Manolio TA, Burke GL, Wolfson SK. Carotidartery intima and media thickness as a risk factor for myocardial infarction and stroke in older adults. Cardiovascular Health Study Collaborative Research Group. N Engl J Med. 1999;340:14-22.

16. Hsue PY. Clinical Features of Acute Coronary Syndromes in Patients With Human Immunodeficiency Virus Infection. Circulation. 2004;109:316-9.

17. Touboul PJ, Hennerici MG, Meairs S, Adams H, Amarenco P, Bornstein N, et al. Mannheim carotid intima-media thickness consensus (2004-2006). An update on behalf of the Advisory Board of the 3rd and 4th Watching the Risk Symposium, 13th and 15th European Stroke Conferences, Mannheim, Germany, 2004, and Brussels, Belgium, 2006. Cerebrovasc Dis. 2007;23:75-80.

18. Perk J, De Backer G, Gohlke H, Graham I, Reiner Z, Verschuren M, et al. European Guidelines on cardiovascular disease prevention in clinical practice (version 2012): The Fifth Joint Task Force of the European Society of Cardiology and Other Societies on Cardiovascular Disease Prevention in Clinical Practice (constituted by representatives of nine societies and by invited experts). Eur Heart J. 2012;33:1635-701.

19. Nixon DE, Landay AL. Biomarkers of immune dysfunction in HIV. Curr Opin HIV AIDS. 2010;5:498-503. function or prothrombotic state contributed to improved assessment of subclinical atherosclerosis. The usefulness of such markers in routine clinical evaluation of HIV infected patients remains unclear and further research is needed.
20. de Gaetano Donati K, Rabagliati R, lacoviello L, Cauda R. HIV infection, HAART, and endothelial adhesion molecules: current perspectives. Lancet Infect Dis. 2004;4:213-22.

21. Wolf K, Tsakiris DA, Weber R, Erb P, Battegay M. Swiss HIV Cohort Study. Antiretroviral therapy reduces markers of endothelial and coagulation activation in patients infected with human immunodeficiency virus type 1. J Infect Dis. 2002;185:456-62.

22. Papasavvas E, Azzoni L, Pistilli M, Hancock A, Reynolds G, Gallo C, et al. Increased soluble vascular cell adhesion molecule-1 plasma levels and soluble intercellular adhesion molecule-1 during antiretroviral therapy interruption and retention of elevated soluble vascular cellular adhesion molecule-1 levels following resumption of antiretroviral therapy. AIDS. 2008;22:1153-61.

23. Noursadeghi M, Miller RF. Clinical value of C-reactive protein measurements in HIV-positive patients. International Journal of STD \& AIDS. 2005;16:438-41.

24. Desvarieux M, Boccara F, Meynard J-L, Bastard J-P, Mallat Z, Charbit B, et al. Infection duration and inflammatory imbalance are associated with atherosclerotic risk in HIV-infected never-smokers independent of antiretroviral therapy. AIDS. 2013;27:2603-14.

25. Baker J, Ayenew W, Quick H, Hullsiek KH, Tracy R, Henry K, et al. High $\square$ density lipoprotein particles and markers of inflammation and thrombotic activity in patients with untreated HIV infection. J Infect Dis. 2010;201:285-92.

26. Lebech A-M, Kristoffersen US, Wiinberg N, Kofoed K, Andersen O, Hesse B, et al. Coronary and peripheral endothelial function in HIV patients studied with positron emission tomography and flow-mediated dilation: relation to hypercholesterolemia. Eur J Nucl Med Mol Imaging. 2008;35:2049-58.

27. Falcão MDCB de A, Zírpoli JC, Albuquerque VM de, Markman Filho B, Araújo NAM de, Falcão CA, et al. Association of biomarkers with atherosclerosis and risk for coronary artery disease in patients with HIV. Arq Bras Cardiol. 2012;99:971 8.

28. Triant VA, Meigs JB, Grinspoon SK. Association of C-reactive protein and HIV infection with acute myocardial infarction. J Acquir Immune Defic Syndr. 2009;51:268-73.

29. De Luca A, de Gaetano Donati K, Colafigli M, Cozzi-Lepri A, De Curtis A, Gori A, et al. The association of high-sensitivity c-reactive protein and other biomarkers with cardiovascular disease in patients treated for HIV: a nested case-control study. BMC Infect Dis. 2013;13:414.

30. Duprez DA, Neuhaus J, Kuller LH, Tracy R, Belloso W, De Wit S, et al. Inflammation, coagulation and cardiovascular disease in HIV-infected individuals. Thorne C, editor. PLoS ONE. 2012;7:e44454.

31. Calza L, Pocaterra D, Pavoni M, Colangeli V, Manfredi R, Verucchi G, et al. Plasma levels of VCAM-1, ICAM-1, E-Selectin, and P-Selectin in 99 HIV-positive patients versus 51 HIV negative healthy controls. J Acquir Immune Defic Syndr. 2009;50:430-2.

32. Francisci D, Giannini S, Baldelli F, Leone M, Belfiori B, Guglielmini G, et al. HIV type 1 infection, and not short-term HAART, induces endothelial dysfunction. AIDS. 2009;23:589-96.

33. Melendez MM, Mcnurlan MA, Mynarcik DC, Khan S, Gelato MC. Endothelial adhesion molecules are associated with inflammation in subjects with HIV disease. Clin Infect Dis. 2008;46:775-80.

34. Kristoffersen US, Kofoed K, Kronborg G, Giger AK, Kjaer A, Lebech AM. Reduction in circulating markers of endothelial dysfunction in HIV-infected patients during antiretroviral therapy. HIV Med. 2009;10:79-87.

35. Guzmán-Fulgencio M, Medrano J, Rallón N, Echeverria-Urabayen A, Benito JM, Restrepo C, et al. Soluble markers of inflammation are associated with Framingham scores in HIV-infected patients on suppressive antiretroviral therapy. J Infect. 2011;63:382-90.

36. Koppel K, Bratt G, Schulman S, Bylund H, Sandström E. Hypofibrinolytic state in HIV-1-infected patients treated with protease inhibitor-containing highly active antiretroviral therapy. J Acquir Immune Defic Syndr. 2002;29:441-9.

37. Grinspoon SK, Grunfeld C, Kotler DP, Currier JS, Lundgren JD, Dube MP, et al. State of the science conference: initiative to decrease cardiovascular risk and increase quality of care for patients living with HIV/AIDS: executive summary. Circulation. 2008;118:198-210. 